International Journal of Pure and Applied Mathematics

Volume 109 No. 3 2016, 673-680

ISSN: 1311-8080 (printed version); ISSN: 1314-3395 (on-line version)

url: http://www.ijpam.eu

doi: 10.12732/ijpam.v109i3.15

\title{
NOVEL IDENTITIES OF SYMMETRY FOR CARLITZ'S TWISTED $q$-BERNOULLI POLYNOMIALS UNDER $S_{4}$
}

\author{
Ugur Duran ${ }^{1 \S}$, Mehmet Acikgoz ${ }^{2}$ \\ ${ }^{1,2}$ Department of Mathematics \\ Faculty of Arts and Science \\ University of Gaziantep \\ TR-27310 Gaziantep, TURKEY
}

\begin{abstract}
In this paper, the authors discover some new symmetric identities of Carlitz's twisted $q$-Bernoulli polynomials arising from the $p$-adic $q$-integral on $\mathbb{Z}_{p}$ under symmetric group of degree four shown by $S_{4}$.
\end{abstract}

AMS Subject Classification: 11S80, 11B68, 05A19, 05A30

Key Words: symmetric identities, Carlitz's twisted $q$-Bernoulli polynomials, $p$-adic $q$ integral on $\mathbb{Z}_{p}$, invariant under $S_{4}$

\section{Introduction}

As well known that the ordinary Bernoulli polynomials, $B_{n}(x)$, are defined by the following Taylor series expansion about $t=0$ :

$$
\sum_{n=0}^{\infty} B_{n}(x) \frac{t^{n}}{n !}=\frac{t}{e^{t}-1} e^{x t}, \quad(|t|<2 \pi) .
$$

Upon setting $x=0$ in the Eq. (1.1), we have $B_{n}(0):=B_{n}$ that is popularly known as $n$-th Bernoulli number (see, e.g., [1], [3], [4], [8], [9], [10], [11]).

Received: $\quad$ Febrauray 4, 2016

Revised: $\quad$ August 16, 2016

Published: September 30, 2016

(c) 2016 Academic Publications, Ltd.

url: www.acadpubl.eu

${ }^{\S}$ Correspondence author 
Imagine that $p$ be a fixed odd prime number. Throughout this paper, $\mathbb{Z}_{p}, \mathbb{Q}$, $\mathbb{Q}_{p}$ and $\mathbb{C}_{p}$ will denote the ring of $p$-adic rational integers, the field of rational numbers, the field of $p$-adic rational numbers and the completion of algebraic closure of $\mathbb{Q}_{p}$, respectively. Let $\mathbb{N}=\{1,2,3, \cdots\}$ and $\mathbb{N}^{*}=\mathbb{N} \cup\{0\}$. The normalized absolute value according to the theory of $p$-adic analysis is given by $|p|_{p}=p^{-1}$. The notation " $q$ " can be considered as an indeterminate, a complex number $q \in \mathbb{C}$ with $|q|<1$, or a $p$-adic number $q \in \mathbb{C}_{p}$ with $|q-1|_{p}<p^{-\frac{1}{p-1}}$ and $q^{x}=\exp (x \log q)$ for $|x|_{p} \leq 1$. The $q$-analogue of $x$ is defined by $[x]_{q}=$ $\left(1-q^{x}\right) /(1-q)$. It is clear that $\lim _{q \rightarrow 1}[x]_{q}=x$ for any $x$ with $|x|_{p} \leq 1$ in the $p$-adic case (for details, see $[1,2,4-12])$.

We say that $f$ is uniformly differentiable function at a point $a \in \mathbb{Z}_{p}$, which is denoted by $f \in U D\left(\mathbb{Z}_{p}\right)$. From here, Kim defined the $q$-Volkenborn integral or $p$-adic $q$-integral on $\mathbb{Z}_{p}$ of a function $f \in U D\left(\mathbb{Z}_{p}\right)$ in [9] as follows:

$$
I_{q}(f)=\int_{\mathbb{Z}_{p}} f(x) d \mu_{q}(x)=\lim _{N \rightarrow \infty} \frac{1}{\left[p^{N}\right]_{q}} \sum_{x=0}^{p^{N}-1} f(x) q^{x} .
$$

If we take $f_{1}(x)=f(x+1)$ in Eq. (1.2), then we see that

$$
q I_{q}\left(f_{1}\right)=I_{q}(f)+(q-1) f(0)+\frac{q-1}{\log q} f^{\prime}(0) .
$$

Also we observe that

$$
q^{n} I_{q}\left(f_{n}\right)=I_{q}(f)+(q-1) \sum_{r=0}^{n-1} q^{r} f(r)+\frac{q-1}{\log q} \sum_{r=0}^{n-1} f^{\prime}(r)
$$

where $f_{n}(x)=f(x+n)$. For these related issues, see, e.g., [2], [4], [5], [6], [7], [8], [9], [10], [11], [12].

Let $T_{p}=\bigcup_{N \geq 1} C_{p^{N}}=\lim _{N \rightarrow \infty} C_{p^{N}}$, where $C_{p^{N}}=\left\{\zeta: \zeta^{p^{N}}=1\right\}$ is the cyclic group of order $p^{N}$. For $\zeta \in T_{p}$, we indicate by $\phi_{\zeta}: \mathbb{Z}_{p} \rightarrow C_{p}$ the locally constant function $x \rightarrow \zeta^{x}$. For $q \in C_{p}$ with $|1-q|_{p}<1$ and $\zeta \in T_{p}$, the Carlitz's twisted $q$-Bernoulli polynomials with Witt's formula are defined by the following $q$-Volkenborn integral on $\mathbb{Z}_{p}$, with respect to $\mu_{q}$, in [11]:

$$
\int_{\mathbb{Z}_{p}} \zeta^{y}[x+y]_{q}^{n} d \mu_{q}(y)=\beta_{n, q, \zeta}(x) \quad(n \geq 0) .
$$


Substituting $x=0$ into the Eq. (1.4) gives $\beta_{n, q, \zeta}(0):=\beta_{n, q, \zeta}$ that are called $n$-th Carlitz's twisted $q$-Bernoulli numbers. The following expression holds

$$
\beta_{n, q, \zeta}(x)=\left([x]_{q}+q^{x} \beta_{q, \zeta}\right)^{n}
$$

with the usual convention of replacing $\beta_{q, \zeta}^{n}$ by $\beta_{n, q, \zeta}$. Taking $\zeta=1$ and $q \rightarrow 1$ in the Eq. (1.4) yields to

$$
\beta_{n, q, \zeta}(x) \rightarrow B_{n}(x):=\int_{\mathbb{Z}_{p}}(x+y)^{n} d \mu_{1}(y),
$$

where $\mu_{1}$ is $p$-adic Haar distribution.

Recently, symmetric identities of some well-known polynomials arising from $p$-adic $q$-integrals on $\mathbb{Z}_{p}$ have been investigated extensively by many mathematicians. For example, Araci et al. [2] investigated some new symmetric identities of $q$-Frobenius polynomials under $S_{5}$, which are associated with the fermionic $p$-adic $q$-integral over the $p$-adic numbers field. Kim et al. [4] derived some novel identities of symmetry for the Carlitz $q$-Bernoulli polynomials invariant under $S_{4}$ which are derived from $p$-adic $q$-integral on $\mathbb{Z}_{p}$. Duran et al. [5] gave some new symmetric identities of $q$-Genocchi polynomials arising from the $q$-Volkenborn integral on $\mathbb{Z}_{p}$ under $S_{4}$. Duran et al. [6] obtained some new symmetric identities of weighted $q$-Genocchi polynomials using $q$-Volkenborn integral on $\mathbb{Z}_{p}$ under $S_{4}$. Duran et al. [7] considered some new symmetric identities of Carlitz's twisted $(h, q)$-Euler polynomials derived from $p$-adic invariant integral on $\mathbb{Z}_{p}$ under $S_{n}$. Moreover, Kim et al. [9] discovered new identities of symmetry for higher-order Carlitz's $q$-Bernoulli polynomials arising from $p$ adic $q$-integral on $\mathbb{Z}_{p}$ under symmetric group of degree five. Furthermore, Kim [10] presented some novel identities of symmetry for Carlitz's-type $q$-Bernoulli polynomials resulting from $p$-adic $q$-integral on $\mathbb{Z}_{p}$ under symmetric group of degree five.

In the next section, we consider the Carlitz's twisted $q$-Bernoulli polynomials and give some novel symmetric identities for these polynomials originating from the $p$-adic $q$-integral on $\mathbb{Z}_{p}$ under symmetric group of degree four shown by $S_{4}$.

\section{Novel identities of symmetry for $\beta_{n, q, \zeta}(x)$ under $S_{4}$}

Let $\zeta \in T_{p}, q \in \mathbb{C}_{p}$ with $|q-1|_{p}<1$ and $w_{i} \in \mathbb{N}$ with $i \in\{1,2,3,4\}$. By the Eqs. (1.2) and (1.4), we discover

$$
\int_{\mathbb{Z}_{p}} \zeta^{w_{1} w_{2} w_{3} y} e^{\left[w_{1} w_{2} w_{3} y+w_{1} w_{2} w_{3} w_{4} x+w_{4} w_{2} w_{3} i+w_{4} w_{1} w_{3} j+w_{4} w_{1} w_{2} k\right]_{q} t} d \mu_{q} w_{1} w_{2} w_{3}(y)
$$




$$
\begin{aligned}
& =\lim _{N \rightarrow \infty} \frac{1}{\left[p^{N}\right]_{q^{w_{1} w_{2} w_{3}}}} \sum_{y=0}^{p^{N}-1} \zeta^{w_{1} w_{2} w_{3} y} q^{w_{1} w_{2} w_{3} y} \\
& \quad \times e^{\left[w_{1} w_{2} w_{3} y+w_{1} w_{2} w_{3} w_{4} x+w_{4} w_{2} w_{3} i+w_{4} w_{1} w_{3} j+w_{4} w_{1} w_{2} k\right]_{q} t} \\
& =\lim _{N \rightarrow \infty} \frac{1}{\left[w_{4} p^{N}\right]_{q_{1} w_{1} w_{2} w_{3}}} \sum_{l=0}^{w_{4}-1} \sum_{y=0}^{p^{N}-1} \zeta^{w_{1} w_{2} w_{3}\left(l+w_{4} y\right)} q^{w_{1} w_{2} w_{3}\left(l+w_{4} y\right)} \\
& \quad \times e^{\left[w_{1} w_{2} w_{3}\left(l+w_{4} y\right)+w_{1} w_{2} w_{3} w_{4} x+w_{4} w_{2} w_{3} i+w_{4} w_{1} w_{3} j+w_{4} w_{1} w_{2} k\right]_{q} t},
\end{aligned}
$$

which yields

$$
\begin{aligned}
I= & \frac{1}{\left[w_{1} w_{2} w_{3}\right]_{q}} \sum_{i=0}^{w_{1}-1} \sum_{j=0}^{w_{2}-1} \sum_{k=0}^{w_{3}-1} \zeta^{w_{4} w_{2} w_{3} i+w_{4} w_{1} w_{3} j+w_{4} w_{1} w_{2} k} \\
& \times q^{w_{4} w_{2} w_{3} i+w_{4} w_{1} w_{3} j+w_{4} w_{1} w_{2} k} \\
& \times \int_{\mathbb{Z}_{p}} \zeta^{w_{1} w_{2} w_{3} y} e^{\left[w_{1} w_{2} w_{3} y+w_{1} w_{2} w_{3} w_{4} x+w_{4} w_{2} w_{3} i+w_{4} w_{1} w_{3} j+w_{4} w_{1} w_{2} k\right]_{q} t} d \mu_{q} w_{1} w_{2} w_{3}(y) \\
= & \lim _{N \rightarrow \infty} \frac{1}{\left[w_{1} w_{2} w_{3} w_{4} p^{N}\right]_{q}} \sum_{i=0}^{w_{1}-1} \sum_{j=0}^{w_{2}-1} \sum_{k=0}^{w_{3}-1} \sum_{l=0}^{w_{4}-1} \sum_{y=0}^{p^{N}-1} \\
& \times \zeta^{w_{1} w_{2} w_{3}\left(l+w_{4} y\right)+w_{4} w_{2} w_{3} i+w_{4} w_{1} w_{3} j+w_{4} w_{1} w_{2} k} \\
& \times q^{w_{1} w_{2} w_{3}\left(l+w_{4} y\right)+w_{4} w_{2} w_{3} i+w_{4} w_{1} w_{3} j+w_{4} w_{1} w_{2} k} \\
& \times e^{\left[w_{1} w_{2} w_{3}\left(l+w_{4} y\right)+w_{1} w_{2} w_{3} w_{4} x+w_{4} w_{2} w_{3} i+w_{4} w_{1} w_{3} j+w_{4} w_{1} w_{2} k\right]_{q} t} .
\end{aligned}
$$

Note that Eq. (2.1) is invariant for any permutation $\sigma \in S_{4}$. Therefore, we acquire the following theorem.

Theorem 1. Let $\zeta \in T_{p}, q \in \mathbb{C}_{p}$ with $|q-1|_{p}<1$ and $w_{i} \in \mathbb{N}$ with $i \in\{1,2,3,4\}$. Then the following

$$
\begin{aligned}
I= & \frac{1}{\left[w_{\sigma(1)} w_{\sigma(2)} w_{\sigma(3)}\right]_{q}} \sum_{i=0}^{w_{\sigma(1)}} \sum_{j=0}^{-1} \sum_{k=0}^{w_{\sigma(2)}-1} w_{\sigma(3)}-1 \\
& \times \zeta^{w_{\sigma(4)} w_{\sigma(2)} w_{\sigma(3)} i+w_{\sigma(4)} w_{\sigma(1)} w_{\sigma(3)} j+w_{\sigma(4)} w_{\sigma(1)} w_{\sigma(2)} k} \\
& \times q^{w_{\sigma(4)} w_{\sigma(2)} w_{\sigma(3)} i+w_{\sigma(4)} w_{\sigma(1)} w_{\sigma(3)} j+w_{\sigma(4)} w_{\sigma(1)} w_{\sigma(2)} k} \\
& \times \int_{\mathbb{Z}_{p}} \exp \left(\left[w_{\sigma(1)} w_{\sigma(2)} w_{\sigma(3)} y+w_{\sigma(1)} w_{\sigma(2)} w_{\sigma(3)} w_{\sigma(4)} x+w_{\sigma(4)} w_{\sigma(2)} w_{\sigma(3)} i\right.\right. \\
& \left.\left.+w_{\sigma(4)} w_{\sigma(1)} w_{\sigma(3)} j+w_{\sigma(4)} w_{\sigma(1)} w_{\sigma(2)} k\right]_{q} t\right)
\end{aligned}
$$




$$
\times \zeta^{w_{\sigma(1)} w_{\sigma(2)} w_{\sigma(3)} y} d \mu_{q} w_{\sigma(1)}{ }^{w_{\sigma(2)} w_{\sigma(3)}}(y)
$$

holds true for any $\sigma \in S_{4}$.

Using the definition of $q$-number, $[x]_{q}$, we easily derive that

$$
\begin{aligned}
& {\left[w_{1} w_{2} w_{3} y+w_{1} w_{2} w_{3} w_{4} x+w_{4} w_{2} w_{3} i+w_{4} w_{1} w_{3} j+w_{4} w_{1} w_{2} k\right]_{q}} \\
& =\left[w_{1} w_{2} w_{3}\right]_{q}\left[y+w_{4} x+\frac{w_{4}}{w_{1}} i+\frac{w_{4}}{w_{2}} j+\frac{w_{4}}{w_{3}} k\right]_{q^{w_{1} w_{2} w_{3}}}
\end{aligned}
$$

From Eq. (2.2), we compute

$$
\begin{aligned}
& \int_{\mathbb{Z}_{p}} \zeta^{w_{1} w_{2} w_{3} y} e^{\left[w_{1} w_{2} w_{3} y+w_{1} w_{2} w_{3} w_{4} x+w_{4} w_{2} w_{3} i+w_{4} w_{1} w_{3} j+w_{4} w_{1} w_{2} k\right]_{q} t} d \mu_{q^{w_{1} w_{2} w_{3}}}(y) \\
& =\sum_{n=0}^{\infty}\left[w_{1} w_{2} w_{3}\right]_{q}^{n}\left(\int _ { \mathbb { Z } _ { p } } \zeta ^ { w _ { 1 } w _ { 2 } w _ { 3 } y } \left[y+w_{4} x+\frac{w_{4}}{w_{1}} i+\frac{w_{4}}{w_{2}} j\right.\right. \\
& \left.\left.+\frac{w_{4}}{w_{3}} k\right]_{q^{w_{1} w_{2} w_{3}}}^{n} d \mu_{q^{w_{1} w_{2} w_{3}}}(y)\right) \frac{t^{n}}{n !}
\end{aligned}
$$

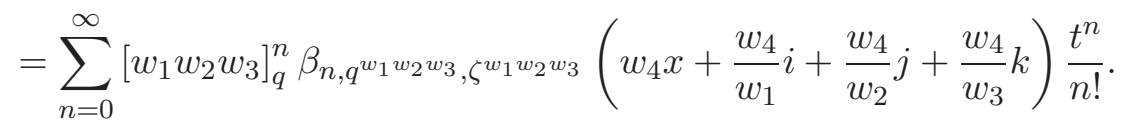

Thus, from Theorem 1 and Eq. (2.3), we conclude the following theorem.

Theorem 2. Let $\zeta \in T_{p}, q \in \mathbb{C}_{p}$ with $|q-1|_{p}<1$ and $w_{i} \in \mathbb{N}$ with $i \in\{1,2,3,4\}$. For $n \geq 0$, the following

$$
\begin{aligned}
& I=\left[w_{\sigma(1)} w_{\sigma(2)} w_{\sigma(3)}\right]_{q}^{n-1} \sum_{i=0}^{w_{\sigma(1)}} \sum_{j=0}^{-1} \sum_{k=0}^{w_{\sigma(2)}-1} \\
& \times \zeta^{w_{\sigma(4)}} w_{\sigma(2)} w_{\sigma(3)} i+w_{\sigma(4)} w_{\sigma(1)} w_{\sigma(3)} j+w_{\sigma(4)} w_{\sigma(1)} w_{\sigma(2)} k \\
& \times q^{w_{\sigma(4)}} w_{\sigma(2)} w_{\sigma(3)} i+w_{\sigma(4)} w_{\sigma(1)} w_{\sigma(3)} j+w_{\sigma(4)} w_{\sigma(1)} w_{\sigma(2)} k
\end{aligned}
$$

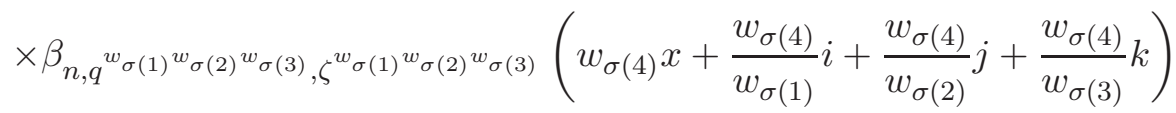

holds true for any $\sigma \in S_{4}$.

Using binomial theorem and the definition of $q$-number, we observe

$$
\left[y+w_{4} x+\frac{w_{4}}{w_{1}} i+\frac{w_{4}}{w_{2}} j+\frac{w_{4}}{w_{3}} k\right]_{q^{w_{1} w_{2} w_{3}}}^{n}
$$




$$
\begin{aligned}
& =\sum_{m=0}^{n}\left(\begin{array}{l}
n \\
m
\end{array}\right)\left(\frac{\left[w_{4}\right]_{q}}{\left[w_{1} w_{2} w_{3}\right]_{q}}\right)^{n-m}\left[w_{2} w_{3} i+w_{1} w_{3} j+w_{1} w_{2} k\right]_{q^{w_{4}}}^{n-m} \\
& \times q^{m\left(w_{2} w_{3} w_{4} i+w_{1} w_{3} w_{4} j+w_{1} w_{2} w_{4} k\right)}\left[y+w_{4} x\right]_{q^{w_{1} w_{2} w_{3}}}^{m},
\end{aligned}
$$

which gives

$$
\begin{aligned}
& {\left[w_{1} w_{2} w_{3}\right]_{q}^{n-1} \sum_{i=0}^{w_{1}-1} \sum_{j=0}^{w_{2}-1} \sum_{k=0}^{w_{3}-1} \zeta^{w_{4} w_{2} w_{3} i+w_{4} w_{1} w_{3} j+w_{4} w_{1} w_{2} k} q^{w_{4} w_{2} w_{3} i+w_{4} w_{1} w_{3} j+w_{4} w_{1} w_{2} k}} \\
& \times \int_{\mathbb{Z}_{p}} \zeta^{w_{1} w_{2} w_{3} y}\left[y+w_{4} x+\frac{w_{4}}{w_{1}} i+\frac{w_{4}}{w_{2}} j+\frac{w_{4}}{w_{3}} k\right]_{q^{w_{1} w_{2} w_{3}}}^{n} d \mu_{q^{w_{1} w_{2} w_{3}}}(y)
\end{aligned}
$$

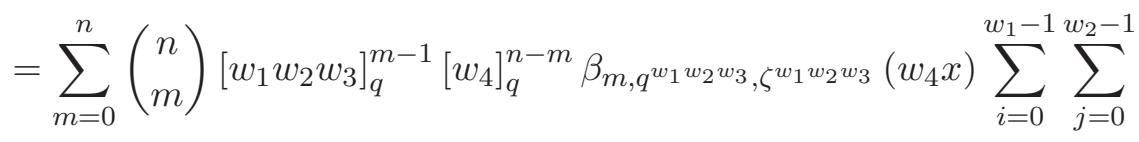

$$
\begin{aligned}
& \sum_{k=0}^{w_{3}-1} \zeta^{w_{4} w_{2} w_{3} i+w_{4} w_{1} w_{3} j+w_{4} w_{1} w_{2} k} q^{(m+1)\left(w_{4} w_{2} w_{3} i+w_{4} w_{1} w_{3} j+w_{4} w_{1} w_{2} k\right)} \\
& \times\left[w_{2} w_{3} i+w_{1} w_{3} j+w_{1} w_{2} k\right]_{q^{w_{4}}}^{n-m}
\end{aligned}
$$

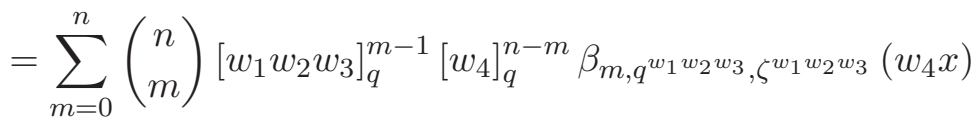

$$
\begin{aligned}
& \times \widehat{C}_{n, q^{w_{4}}, \zeta^{w_{4}}}\left(w_{1}, w_{2}, w_{3} \mid m\right),
\end{aligned}
$$

where

$$
\begin{aligned}
& \widehat{C}_{n, q, \zeta}\left(w_{1}, w_{2}, w_{3} \mid m\right) \\
& =\sum_{i=0}^{w_{1}-1} \sum_{j=0}^{w_{2}-1} \sum_{k=0}^{w_{3}-1} \zeta^{w_{2} w_{3} i+w_{1} w_{3} j+w_{1} w_{2} k} \\
& \times q^{(m+1)\left(w_{2} w_{3} i+w_{1} w_{3} j+w_{1} w_{2} k\right)}\left[w_{2} w_{3} i+w_{1} w_{3} j+w_{1} w_{2} k\right]_{q}^{n-m} .
\end{aligned}
$$

Therefore, by (2.6), we arrive at the following theorem.

Theorem 3. Let $\zeta \in T_{p}, q \in \mathbb{C}_{p}$ with $|q-1|_{p}<1$, wi $\in \mathbb{N}$ with $i \in$ $\{1,2,3,4\}$, and let $n \geq 0$. Then the following expression

$$
\begin{aligned}
& \sum_{m=0}^{n}\left(\begin{array}{l}
n \\
m
\end{array}\right)\left[w_{\sigma(1)} w_{\sigma(2)} w_{\sigma(3)}\right]_{q}^{m-1}\left[w_{\sigma(4)}\right]_{q}^{n-m}
\end{aligned}
$$

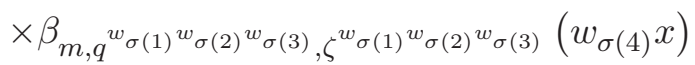




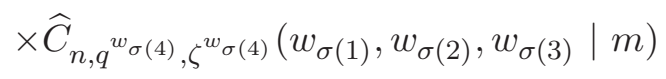

holds true for some $\sigma \in S_{4}$.

\section{Conclusion}

In this study, we have investigated not only new but also interesting symmetric identities for Carlitz's twisted $q$-Bernoulli polynomials arising from the $p$-adic $q$-integral on $\mathbb{Z}_{p}$ under the symmetric group of degree four. We note that in the case $\zeta=1$, the results derived in this paper reduce to the results in [4].

\section{References}

[1] M. Acikgoz, S. Araci, U. Duran, New extensions of some known special polynomials under the theory of multiple q-calculus, Turkish Journal of Analysis and Number Theory, Vol. 3, No. 5, (2015) pages 128-139, doi: 10.12691/tjant-3-5-4

[2] S. Araci, U. Duran, M. Acikgoz, Symmetric identities involving q-Frobenius-Euler polynomials under Sym (5), Turkish Journal of Analysis and Number Theory, Vol. 3, No. 3, pp. 90-93 (2015), doi: 10.12691/tjant-3-3-5

[3] S. Araci, M. Acikgoz, A. Bagdasaryan, E. Sen, The Legendre Polynomials Associated with Bernoulli, Euler, Hermite and Bernstein Polynomials, Turkish Journal of Analysis and Number Theory, Vol. 1, No. 1, (2013) 1-3, doi: 10.12691/tjant-1-1-1

[4] D. S. Kim, T. Kim, Some identities of symmetry for Carlitz q-Bernoulli polynomials invariant under $S_{4}$, Ars Combinatoria, Vol. 123. (2015) pp. 283-289.

[5] U. Duran, M. Acikgoz, A. Esi, S. Araci, Some new symmetric identities involving qGenocchi polynomials under $S_{4}$, Journal of Mathematical Analysis, Vol 6, Issue 4, (2015) pages 22-31.

[6] U. Duran, M. Acikgoz, S. Araci, Symmetric identities involving weighted q-Genocchi polynomials under $S_{4}$, Proceedings of the Jangjeon Mathematical Society,18, No. 4, (2015) pp 455-465, doi: 10.13140/RG.2.1.1723.6648

[7] U. Duran, M. Acikgoz, New identities for Carlitz's twisted (h, $q$ )-Euler polynomials under symmetric group of degree $n$, Journal of Analysis and Number Theory, Vol. 4, No. 2, (2016) 133-137, doi: 10.18576/jant/040208

[8] T. Kim, q-Volkenborn Integration, Russian Journal of Mathematical Physics 9.3, (2002) pp. 288-299.

[9] T. Kim, Some new identities of symmetry for higher-order Carlitz q-Bernoulli polynomials arising from p-adic q-integral on $\mathbb{Z}_{p}$ under the symmetric group of degree five, Applied Mathematical Sciences, Vol. 9, no. 93, (2015) 4627-4634.

http://dx.doi.org/10.12988/ams.2015.5340 
[10] T. Kim, J. J. Seo, New identities of symmetry for Carlitz's-type q-Bernoulli polynomials under symmetric group of degree five, International Journal of Mathematical Analysis, Vol. 9, no. 35, (2015) 1707 - 1713.

http://dx.doi.org/10.12988/ijma.2015.53110

[11] C. S. Ryoo, Symmetric identities for Carlitz's twisted q-Bernoulli polynomials associated with p-adic q-integral on $\mathbb{Z}_{p}$, Applied Mathematical Sciences, Vol. 9, no. 72, (2015) 3569-3575.

http://dx.doi.org/10.12988/ams.2015.53225

[12] J. J. Seo, T. Kim, Identities of symmetry for generalized q-Euler polynomials attached to $\chi$ under symmetric group $S_{4}$, Advanced Studies in Theoretical physics, Vol. 9, no. 8, (2015) 353-359.

http://dx.doi.org/10.12988/astp.2015.5227 